\title{
Primary non-gestational pure choriocarcinoma arising in the ovary: A case report and literature review
}

\author{
SHINICHI HAYASHI ${ }^{1}$, YOSHIRO ABE ${ }^{2}$, SHIGEKI TOMITA ${ }^{3}$, YUKO NAKANISHI ${ }^{1}$, \\ SHIGEHARU MIWA ${ }^{1}$, TAKAHIKO NAKAJIMA ${ }^{1}$, KAZUHIRO NOMOTO ${ }^{1}$, \\ KOICHI TSUNEYAMA ${ }^{1}$, TAKAHIRO FUJIMORI ${ }^{3}$ and JOHJI IMURA ${ }^{1}$ \\ ${ }^{1}$ Department of Diagnostic Pathology, Graduate School of Medicine and Pharmaceutical Sciences, University of Toyama, \\ Toyama, Toyama 930-0194; Departments of ${ }^{2}$ Neurosurgery and ${ }^{3}$ Surgical and Molecular Pathology, \\ Dokkyo University School of Medicine, Tochigi 321-0293, Japan
}

Received December 23, 2013; Accepted October 20, 2014

DOI: $10.3892 / \mathrm{ol} .2015 .2985$

\begin{abstract}
Pure non-gestational choriocarcinoma is a primary germ cell neoplasm that has been defined as a tumor without other germ cell elements. The current study presents an extremely rare case of non-gestational pure choriocarcinoma in a postmenarcheal young female and describes details of the tumor, including the clinicopathological findings. The patient was a 10 -year-old female who underwent salpingo-oophorectomy. Histologically, the extensive hemorrhagic tumor was composed of choriocarcinoma without additional germ cell tumor components. The tumor cells were immunohistochemically positive for epithelial markers, including cytokeratins and epithelial membrane antigens, and there was a positive cytoplasmic reaction for $\beta$-human chorionic gonadotropin in the syncytiotrophoblasts. Furthermore, numerous tumor cells were immunohistochemically positive for the 32 -microglobulin antibody. The patient received adjuvant cisplatin, etoposide and bleomycin chemotherapy, and is currently disease-free without evidence of recurrence or metastasis subsequent to 62 months of follow-up.
\end{abstract}

\section{Introduction}

A malignant tumor of the ovary with trophoblastic differentiation may be a gestational or non-gestational choriocarcinoma and may be a primary tumor or a metastasis from other organs. Predominantly, non-gestational choriocarcinoma occurs as a component of a mixed germ cell tumor $(1,2)$.

Correspondence to: Professor Johji Imura, Department of Diagnostic Pathology, Graduate School of Medicine and Pharmaceutical Sciences, University of Toyama, 2,630 Sugitani, Toyama, Toyama 930-0194, Japan

E-mail: imura@med.u-toyama.ac.jp

Key words: choriocarcinoma, non-gestational, ovary, $\beta 2$-microglobulin, pathology
Pure non-gestational choriocarcinoma is an extremely rare primary germ cell neoplasm, with reports of only a few cases at the time of the present case $(3,4)$. Pure choriocarcinoma has been defined as a tumor that does not include other germ cell tumor elements (1). It is necessary to distinguish between cases of non-gestational or gestational choriocarcinoma of the ovary, as cases of non-gestational choriocarcinoma have a poorer prognosis compared with gestational cases, and the treatment regimen for the two diseases differs. In general, the non-gestational type has only been clinically diagnosed in patients who were sexually immature, unable to conceive or had never had sexual intercourse (5). In the absence of clinical information, it is difficult to differentiate the two diseases morphologically. The current study reports a rare case of non-gestational pure choriocarcinoma in a postmenarcheal young female and describes details of the tumor, including the clinicopathological findings. Furthermore, a differential diagnosis between non-gestational and gestational choriocarcinoma of the ovary, based on immunohistochemical differences, is discussed. Consent was obtained from the family of the patient.

\section{Case report}

In June 2004, a 10-year-old Japanese female presented to Dokkyo University School Hospital (Tochigi, Japan) with abdominal pain and a mass. The patient had experienced diarrhea alternating with constipation and low-grade fever for the previous two months. Furthermore, weight loss of $8 \mathrm{~kg}$ had been exhibited over the previous three months, with decreased food intake. The patient experienced the first menarche six months prior to presenting, and the periods of menstruation were irregular. The medical history and the family history were non-contributory.

Upon physical examination, an uneven, firm mass (20 $\mathrm{cm}$ in diameter) was palpable in the abdomen. The remaining systemic examination was unremarkable. The serum $\beta$-human chorionic gonadotropin (hCG) and serum $\alpha$-fetoprotein levels were $6,600 \mathrm{ng} / \mathrm{ml}$ (normal, $<0.1 \mathrm{ng} / \mathrm{ml}$ ) and $<5 \mathrm{ng} / \mathrm{ml}$ (normal, $<10 \mathrm{ng} / \mathrm{ml}$ ), respectively. Magnetic resonance imaging showed the pelvic mass, which exhibited 
Table I. Clinicopathological characteristics of 32 cases of non-gestational pure ovarian choriocarcinoma.

\begin{tabular}{lc}
\hline Variable & Value \\
\hline Mean age, years & $13.8 \pm 4.2$ \\
Duration of abdominal pain, weeks & $3-16$ \\
Side, \% & \\
Right & 68 \\
Left & 32 \\
Stage, \% & \\
I & 49 \\
II & 17 \\
III & 17 \\
IV & 17
\end{tabular}

Surgery, \%

$\mathrm{UO} / \mathrm{SO}$

$\mathrm{AH}+\mathrm{BSO}$

BSO

20

$\mathrm{hCG}, \mathrm{mU} / \mathrm{ml}$

$0.034-200,000$

Pregnancy test, $\mathrm{n}$

Treatment, $\mathrm{n}$

Bone marrow transplant

Chemotherapy

Methotrexate-based

Vinblastin, bleomycin and cisplatin

Cisplatin, etoposide and bleomycin

Outcome, $\%$

Succumbed

Alive

$\mathrm{UO} / \mathrm{SO}$, unilateral oophorectomy/salpingo-oophorectomy; hCG, human chorionic gonadotropin; BSO, bilateral salpingo-oophorectomy; $\mathrm{AH}$, abdominal hysterectomy.

high intensity with a septal structure on T1-weighted images and irregular high intensity on T2-weighted images, with peripheral enhancement for gadolinium-diethylenetriamine pentaacetic acid.

The patient was diagnosed as having an ovarian tumor and underwent a celiotomy. Intraoperatively, bloody ascitic fluid was observed in the peritoneal cavity, together with a dark red fudge-like and fragile $18 \times 15 \times 10-\mathrm{cm}$ mass, weighing $1,110 \mathrm{~g}$ and involving the right ovary. A small number of tumor nodules were disseminated in the Douglas pouch. The intraperitoneal fudge-like mass was macroscopically resected by right salpingo-oophorectomy.

Upon gross inspection, the mass predominantly consisted of hemorrhagic coagulation necrosis, however, viable areas were observed in certain regions of the tumor (Fig. 1). Histological specimens were randomly prepared from 80 sections taken from various sites, followed by routine staining and

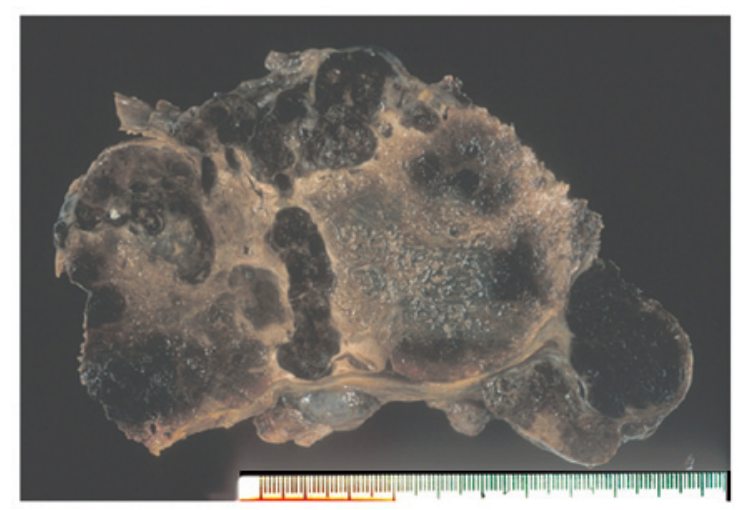

Figure 1. Fudge-like and fragile mass revealing extensive hemorrhagic necrosis.

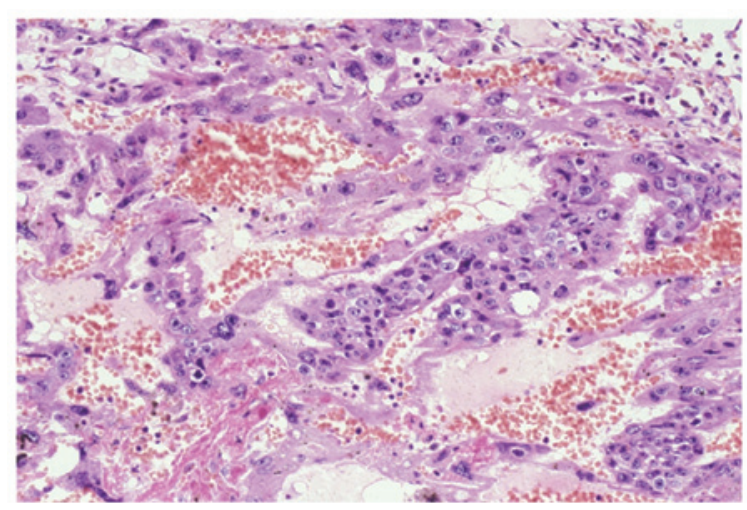

Figure 2. Tumor showing the biphasic appearance of mononuclear cytotrophoblasts and multinucleated syncytiotrophoblasts with hemorrhage.

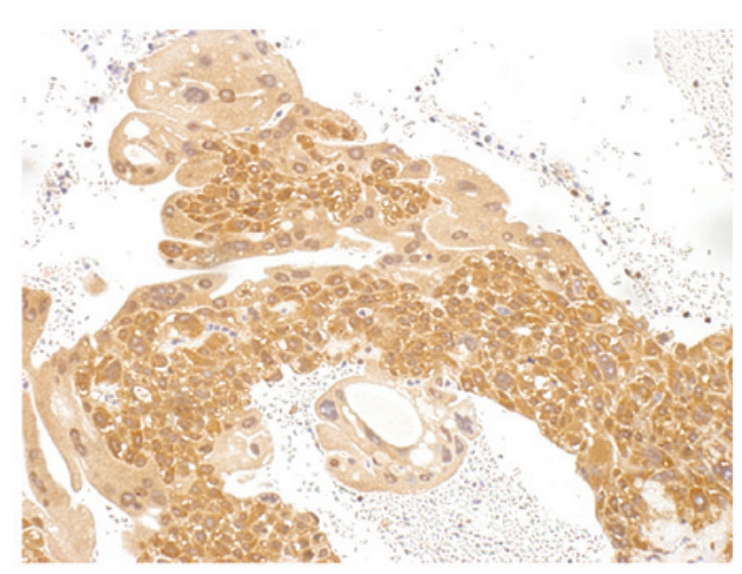

Figure 3. Positive immunoreactivity for $\beta 2$-microglobulin in the cytotrophoblast and syncytiotrophoblast tumor cells.

immunohistochemistry. Microscopically, viable tumor cells were present in the periphery of the mass and were aggregated with one another. The tumor cells were characterized by an intimate admixture of two cell types, mononuclear and multinuclear giant cells, which occasionally exhibited a plexiform pattern. The mononuclear cells were medium in size, polygonal or round, with clear or amphophilic cytoplasm, and exhibited a well-defined cell border. The nuclei of these cells were round and hyperchromatic, with conspicuous nucleoli. Numerous 
mitotic cells were observed. These findings indicated cytotrophoblastic cells. Conversely, the multinuclear giant cells varied in shape and size, and were irregularly-shaped cells with abundant dense amphophilic or vacuolated cytoplasm, and multiple hyperchromatic nuclei without mitosis. These multinuclear cells were regarded as syncytiotrophoblasts (Fig. 2). No evidence of other germ cell elements or chorionic stroma was observed. Immunohistochemically, although positive cytoplasmic staining was observed in the syncytiotrophoblasts for $\beta$-hCG (rabbit polyclonal antibody; Nichirei, Tokyo, Japan), no reaction was observed in the cytotrophoblasts. Additionally, positive staining was observed for epithelial markers, such as cytokeratin AE1/AE3 (AE1+AE3; Dako, Carpinteria, CA, USA) and epithelial membrane antigen (E29; Dako) in the two cell types. Furthermore, the cells were also histochemically positive for the $\beta 2$-microglobulin (BMG) antibody (rabbit polyclonal antibody; Dako) (Fig. 3).

One week after surgery, the patient received three cyles ,each lasting three weeks, of chemotherapy: cisplatin (33 $\mathrm{mg} / \mathrm{m}^{2}$, days $1-3$ ), etoposide $\left(167 \mathrm{mg} / \mathrm{m}^{2}\right.$, days $\left.1-3\right)$ and bleomycin $\left(15 \mathrm{U} / \mathrm{m}^{2}\right.$, day 1) (PEB). The post-therapeutic period was favorable, with no major complications. The serum hCG level returned to normal $(0.1 \mathrm{ng} / \mathrm{ml})$ five months after the PEB chemotherapy. Furthermore, the patient is currently disease-free without evidence of recurrence or metastasis subsequent to 62 months of follow-up.

\section{Discussion}

Two forms of ovarian choriocarcinoma, gestational and non-gestational, have been reported. Gestational choriocarcinoma of the ovary may be the result of metastasis from a uterine trophoblastic disease, or, rarely, it may follow an ovarian pregnancy. The non-gestational type is a primary germ cell neoplasm differentiating along the extraembryonic chorionic tissue. The majority of the tumors occur in combination with other germ cell neoplasms. Pure choriocarcinoma of the ovary has been defined as a tumor that occurs in the absence of all other germ cell tumors. No additional components of germ cell tumors were found in association with the present tumor. Furthermore, the $\alpha$-fetoprotein levels were normal. Pure ovarian choriocarcinoma is extremely rare $(1,2)$, and based on an extensive search of the English literature, it can be observed that only a few cases of this type of choriocarcinoma have been previously described (Table I) $(3,4)$. This tumor most commonly occurs in young individuals, and $\sim 50 \%$ of cases are identified at a relatively early stage. In addition, in $\sim 66 \%$ of patients chemotherapy improves prognosis.

The present case exhibited the required pathological characteristics, as described in the previously reported cases, to determine a diagnosis of pure choriocarcinoma (1). No other carcinomatous or germ cell tumor elements were identified in addition to the trophoblastic cells. Furthermore, the histological examination revealed no similar tumors in other organs. However, it was difficult to determine morphologically whether the case was a non-gestational or gestational tumor. The majority of studies have suggested that the diagnosis of pure non-gestational choriocarcinoma should be limited to premenarcheal patients $(1-3,5)$.
Differentiation between non-gestational and gestational choriocarcinoma is required, as the chemotherapy and prognosis differ for the two tumor types. A study of the tumor characteristics has been previously performed in vitro by the genetic analysis of cell lines. Tanaka et al (6) reported a lack of effective messenger RNA for BMG in gestational human choriocarcinoma cell lines, as well as the presence of messenger RNA for BMG in non-gestational choriocarcinoma. Several previous studies have also investigated the high expression levels of BMG in choriocarcinoma. Kato et al (7) investigated choriocarcinoma cells that produced moderate amounts of surface and secreted BMG. Norman et al (8) also reported that the serum BMG level was elevated in patients with choriocarcinoma. The study indicated that BMG may be clinically used as a serum marker for this disease. In the present study, BMG was detected in each of the two types of tumor cells using immunohistochemistry. By contrast, there have been no previous studies to immunohistochemically confirm the expression of BMG in the trophoblastic tumor; to the best of our knowledge, the current case study is the first to present this point. Based on the immunohistochemical findings and previous studies, a diagnosis of primary, pure non-gestational choriocarcinoma of the ovary was suggested. This study indicates that BMG may be clinically used as a serum marker for non-gestational choriocarcinoma in the future. However, the cause of the BMG expression in non-gestational choriocarcinoma remains unclear.

In conclusion, the current study presents a case of primary pure ovarian choriocarcinoma, which was successfully treated with surgery and a PEB regimen. Further pathological and biological techniques for the differentiation of gestational and non-gestational tumors is required for the efficient treatment of choriocarcinoma in the future.

\section{References}

1. Russell P and Farnsworth A (eds): Non-gestational choriocarcinomas. In: Surgical Pathology of the Ovaries. 2nd edition. Churchill Livingstone, Edinburgh, pp263-264, 1997.

2. Scully RE, Young RH and Clement PB (eds): Choriocarcinoma. In: Tumors of the Ovary, Maldeveloped Gonads, Fallopian Tube, and Broad Ligament. Atlas of Tumor Pathology. 3rd series. Fascicle 23. Armed Forces Institute of Pathology, Washington DC, pp258-260, 1998.

3. Goswami D, Sharma K, Zutshi V, Tempe A and Nigam S: Nongestational pure ovarian choriocarcinoma with contralateral teratoma. Gynecol Oncol 80: 262-266, 2001.

4. Inaba H, Kawasaki H, Hamazaki M, et al: A case of metastatic ovarian non-gestational choriocarcinoma: successful treatment with conservative type surgery and myeloablative chemotherapy. Pediatr Int 42: 383-385, 2000.

5. Jacobs AJ, Newland JR and Green RK: Pure choriocarcinoma of the ovary. Obstet Gynecol Surv 37: 603-609, 1982.

6. Tanaka K, Nabeshima Y, Takahashi H, et al: Lack of effective messenger RNA for beta 2-microglobulin in a gestational human choriocarcinoma cell line (GCH-1). Cancer Res 41: 3639-3641, 1981

7. Kato M, Ohashi K, Saji F, Wakimoto A and Tanizawa O: Expression of HLA class I and beta 2-microglobulin on human choriocarcinoma cell lines: induction of HLA class I by interferon-gamma. Placenta 12: 217-226, 1991.

8. Norman RJ, Jialal I, Joubert SM and Green-Thompson RW: Beta-2-microglobulin in trophoblastic disease. S Afr Med J 64: 90-92, 1983. 